\title{
Role of the Nuclear Receptor Corepressor 1 (NCOR1) in Atherosclerosis and Associated Immunometabolic Diseases
}

\author{
Martin A. Geiger ${ }^{1}$, Ana T. Guillaumon ${ }^{1}$, Francesco Paneni ${ }^{2,3,4}$, Christian M. Matter ${ }^{2,3}$ \\ and Sokrates Stein ${ }^{2,3 *}$ \\ 1 Vascular Diseases Discipline, Clinics Hospital of the University of Campinas, Campinas, Brazil, ${ }^{2}$ Center for Molecular \\ Cardiology, University of Zurich, Schlieren, Switzerland, ${ }^{3}$ Department of Cardiology, University Heart Center, University \\ Hospital Zurich, Zurich, Switzerland, ${ }^{4}$ Department of Research and Education, University Hospital Zurich, Zurich, Switzerland
}

OPEN ACCESS

Edited by:

Martin Giera,

Leiden University Medical Center,

Netherlands

Reviewed by:

Bart Everts,

Leiden University Medical Center,

Netherlands

Nicolle Kraenkel,

Charité-Universitätsmedizin

Berlin, Germany

*Correspondence:

Sokrates Stein

sokrates.stein@me.com

Specialty section:

This article was submitted to

Cytokines and Soluble

Mediators in Immunity,

a section of the journal

Frontiers in Immunology

Received: 03 June 2020

Accepted: 24 September 2020

Published: 08 October 2020

Citation:

Geiger MA, Guillaumon AT, Paneni F, Matter CM and Stein S (2020) Role of the Nuclear Receptor

Corepressor 1 (NCOR1) in

Atherosclerosis and Associated Immunometabolic Diseases.

Front. Immunol. 11:569358.

doi: 10.3389/fimmu.2020.569358
Atherosclerotic cardiovascular disease is part of chronic immunometabolic disorders such as type 2 diabetes and nonalcoholic fatty liver disease. Their common risk factors comprise hypertension, insulin resistance, visceral obesity, and dyslipidemias, such as hypercholesterolemia and hypertriglyceridemia, which are part of the metabolic syndrome. Immunometabolic diseases include chronic pathologies that are affected by both metabolic and inflammatory triggers and mediators. Important and challenging questions in this context are to reveal how metabolic triggers and their downstream signaling affect inflammatory processes and vice-versa. Along these lines, specific nuclear receptors sense changes in lipid metabolism and in turn induce downstream inflammatory and metabolic processes. The transcriptional activity of these nuclear receptors is regulated by the nuclear receptor corepressors (NCORs), including NCOR1. In this review we describe the function of NCOR1 as a central immunometabolic regulator and focus on its role in atherosclerosis and associated immunometabolic diseases.

Keywords: atherosclerosis, cardiometabolic, corepressor complex, NCoR1, nuclear receptor signaling, immunometabolism, mechanisms of disease, transcriptional regulation

\section{INTRODUCTION}

Atherosclerosis is characterized by the accumulation of immune cells, cholesterol species and other lipids in the intimal space of arteries. The disease primarily affects large, elastic, and high-pressure vessels, such as the coronary, renal, femoral, and carotid arteries. The complex pathophysiology is triggered by genetic and environmental risk factors, including lipid species and metabolites (e.g., cholesterol, specific fatty acids, carnitine), as well as by hypertension, diabetes and obesity (1-3). Molecular, genetic, dietary and pharmacological studies over the last decades suggest that hyperlipidemia, especially hypercholesterolemia, combined with the genetic predisposition is a major trigger of atherogenesis (4-9). Nevertheless, recent research showed that other metabolic and inflammatory processes are closely interconnected at the cellular level as well as via intra- and interorgan communication (10-12) (Figure 1). 


\section{IMMUNOMETABOLIC INTEGRATORS}

The signaling cascades that are activated by inflammatory and metabolic triggers and/or mediators converge at key transcriptional regulators, which in turn coordinate the expression of specific target genes and atherogenic processes. Whether individual target genes are activated or repressed depends on several other factors, such as folding and compaction of the chromatin, posttranslational modifications of histones by chromatin-modifying enzymes, functional alterations by noncoding RNAs, and recruitment of the transcriptional machinery, including transcription factors and importantly also transcription cofactors, i.e. transcriptional corepressors and coactivators (13-16).

\section{Lipid-Responsive Nuclear Receptors With Immunometabolic Functions}

Nuclear receptors are a large family of druggable transcription factors that exert important functions in development, metabolism, and immune response $(17,18)$. Consequently, several nuclear receptors do affect the development of atherosclerosis and associated cardiometabolic diseases (reviewed in (19)). While the role of lipid-binding nuclear receptor in metabolism is well established, studies over the last decade demonstrated that several of these nuclear receptors, including PPAR $\gamma$, LXRs, and LRH-1, mediate transrepression of pro-inflammatory molecules in the liver and/or immune cells, such as macrophages and $\mathrm{T}$ cells (20-25). Therefore, these nuclear receptors act as direct immunometabolic regulators and play an important role in atherogenesis.

\section{NCOR1-An Emerging Regulator of Immunometabolic Processes}

Given their central role to integrate upstream information and regulate the expression of downstream target genes, specific transcriptional cofactors function as central immunometabolic regulators. About 300 transcription cofactors are known to exist in mice and human cells (26). However, only a fraction of those is expressed in a specific cell type or tissue, and their function is restricted to certain pathways and transcription factors $(14,15)$. Some of these factors are involved in inflammatory mechanisms, others known to exert metabolic functions.
Could research on (anti-)atherogenic corepressor complexes provide answers to the key question of causality between metabolic and inflammatory changes? One large nuclear receptor corepressor complex that has been studied extensively in vitro and in vivo in the last years is nuclear receptor corepressor 1 (NCOR1) (Figure 2).

NCOR1 serves as scaffolding protein that forms the basis for a large corepressor complex, including protein deacetylases [including class I (HDAC3), class II (HDAC4, 5, 7, and 9) and class III (SIRT1) KDACs], transducin beta-like 1 (TBL1) and TBLR1, two highly related $\mathrm{F}$ box/WD40-containing factors, and the G-protein-pathway suppressor 2 (GPS2) $(27,28)$. The molecular functions and (patho-) physiological role of HDAC3, class II HDACs, GPS2, and TBL1 has been extensively reviewed (29-31). Although germline $\mathrm{Ncorl}^{-1-}$ and $\mathrm{Ncor2}^{-/-}$mice are embryonically lethal $(32,33)$, they enabled to establish important roles for NCOR1 in erythropoiesis, T-cell, and central nervous system development, whereas NCOR2 contributes to the morphological development of the heart (32-34). Targeted deletions of NCOR1 in immune cells, liver, adipose tissue, and muscle demonstrated that it affects pro- and anti-inflammatory gene signatures, mitochondrial function, lipid metabolism, insulin sensitivity, intestinal cholesterol absorption, thereby highlighting its immunometabolic functions, which will be discussed below (21, 35-41). To the best of our knowledge there are no studies addressing the tissue-specific deletion of Ncor2, which is essential to study the cell-specific function of a gene, especially for genes that are expressed in nearly all cells and organs and thus regulate multiple processes (e.g., a detailed expression pattern of Ncor1 and Ncor2 in mice can be found at Tabula muris (https:// tabula-muris.ds.czbiohub.org). Therefore, this review will focus on tissue-specific functions of NCOR1.

\section{NCOR1 Complex in Macrophages}

Monocyte-derived macrophages are the most abundant immune cell population found in atherosclerotic lesions and plaques. They take up oxidized LDL (oxLDL) in an uncontrolled fashion, secrete pro-inflammatory cytokines that recruit $\mathrm{T}$ cells, and fall apart in a necrotic fashion, thus promoting plaque growth and destabilization $(42,43)$. Silencing of macrophage NCOR1 in vitro produces the same phenotype of activated

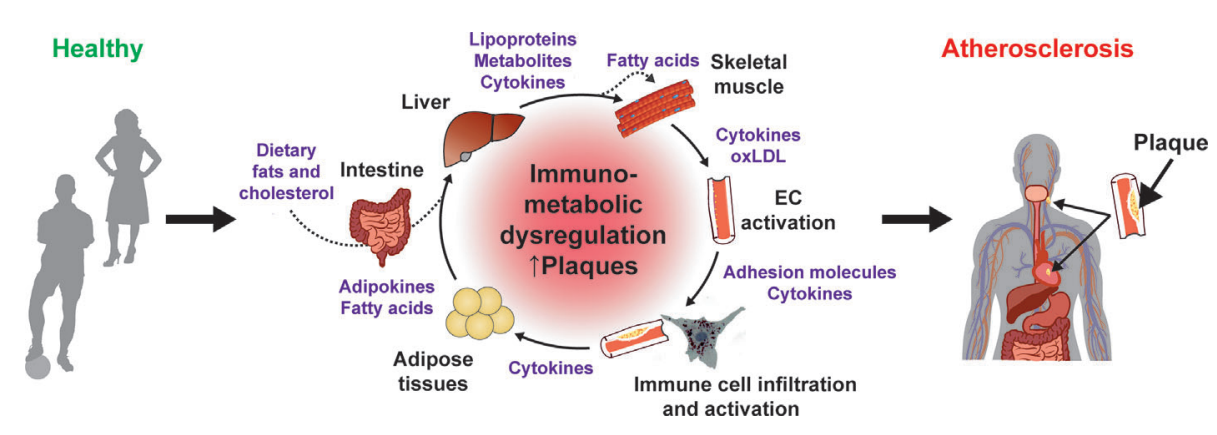

FIGURE 1 | Immunometabolic dysregulation promotes atherogenesis. Contribution of different organs and immunometabolic mediators to the chronic dysregulation that promote atherogenesis. 


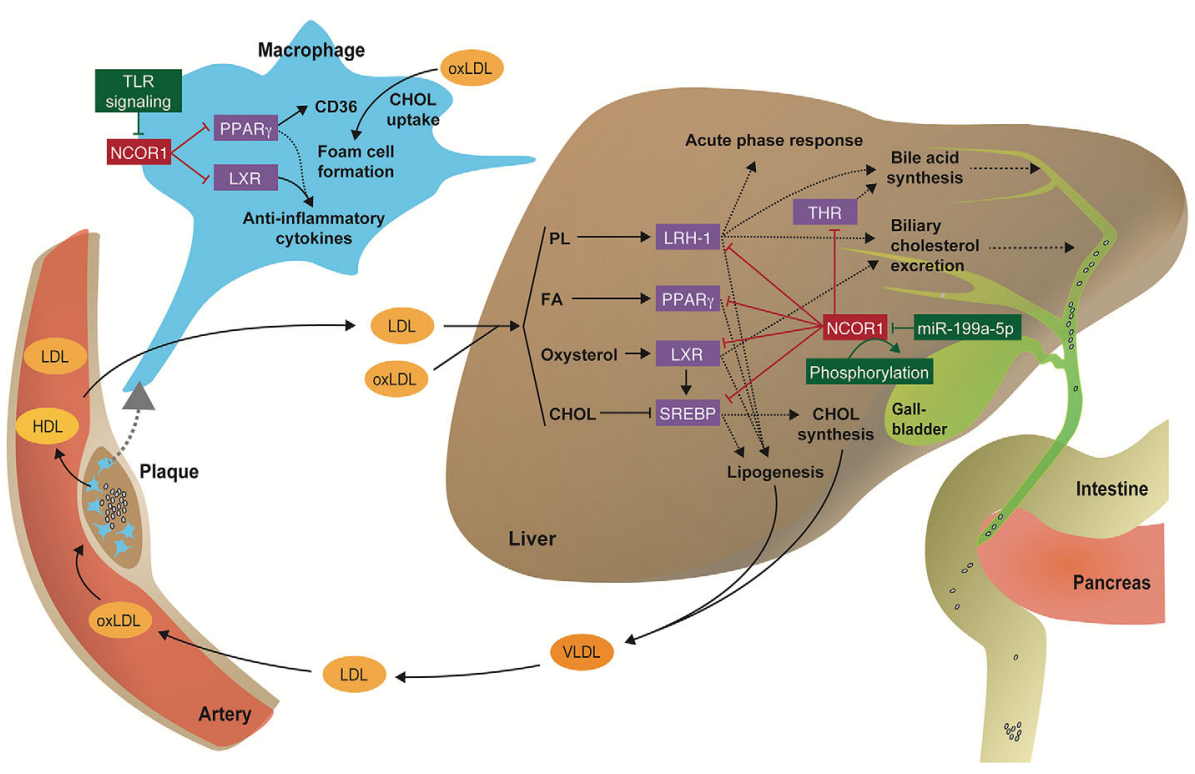

FIGURE 2 | Regulation of immunometabolic processes by the NCOR1 complex. Scheme illustrating how transcriptional corepressor complexes repress the function of nuclear receptors and thus regulate key inflammatory and metabolic processes in the liver and macrophages that are involved in the pathophysiology of cardiometabolic diseases. CHOL, cholesterol; FA, fatty acid; oxLDL, oxidized LDL; PL, phospholipid.

macrophages: increased expression pro-inflammatory cytokines, chemokines and metalloproteases, and enhanced macrophage invasiveness (21). Mechanistically, it was proposed that the NCOR1 corepressor complex acts as a transcriptional checkpoint for these inflammatory genes: In the absence of a nuclear receptor ligand, NCOR1 is recruited to target genes and interacts with unphosphorylated c-Jun bound to target promoters, thus repressing pro-inflammatory gene expression. Upon stimulation with an innate inflammatory stimulus, such as LPS, a structural change in the ligand-binding domain of the nuclear receptor reduces its affinity to NCOR1, hence simultaneously increasing the affinity to coactivators (44). TBLR1 plays a crucial function as an E3-ligase that directs the ligand-dependent ubiquitination and proteasome-mediated clearance of NCOR1 and histone deacetylase 3 (HDAC3) from the promoter of target genes (45). Moreover, proatherosclerotic minimally oxidized LDL (oxLDL) promotes JNKdependent derepression of AP-1 by releasing NCOR1 from the promoter of chemokine genes (40). Taken together, these in vitro studies suggest that absence of NCOR1 exacerbates the inflammatory response in macrophages.

Paradoxically, the in vivo myeloid cell-specific deletion of Ncor1 repressed inflammatory gene expression and improved insulin sensitivity in a diet-induced obesity mouse model (39). Li et al. suggest that deletion of Ncorl in adipose tissue macrophages leads to a derepression of LXR target genes, increased expression of de novo lipogenesis and fatty acid desaturation genes, and subsequent production of anti-inflammatory $\omega 3$ fatty acids, which in turn suppress inflammatory activation of macrophages (39) (Figure 2).

Using a similar loss-of-function approach, Oppi et al. recently showed that the myeloid cell-specific deletion of Ncor1 increased atherosclerosis at the aortic sinus and the thoraco-abdominal aorta in Ldlr knockout mice (41). At the molecular level NCOR1 was found to bind to the Cd36 promoter thus repressing PPAR $\gamma$ driven Cd36 expression. Consequently, peritoneal macrophages from Ncor1-deficient mice had increased CD36-mediated oxLDL accumulation and foam cell formation (Figure 2). Interestingly, Ncor1-deficient macrophages displayed an increased expression of both anti- and pro-inflammatory genes. Moreover, analyses of omics-datasets obtained from human plaque specimens suggested that NCOR1-driven PPAR $\gamma$ suppression is also protective in human plaque development and vulnerability (41).

The data from Oppi et al. and Li et al. are partially controversial $(39,41)$. Two major reasons could explain the differences. First, the genetic background of the mice: Myeloid cell-specific Ncor1 knockout mice on a 'wildtype' versus Ldlr-/- background. The Ldlr deficiency shifts the lipoprotein metabolism towards a humanized profile with very high LDL-cholesterol levels, which makes this mouse model prone to develop atherosclerosis and display a pro-inflammatory signature $(9,46)$. Second, the use of the experimental diets: High-fat diet to study the impact of NCOR1 on diet-induced obesity versus high-cholesterol diet to assess atherogenesis. It is known from numerous studies that diets differently affect major immune and metabolic processes and hence the development of chronic immunometabolic diseases, especially when investigating lipid-sensitive nuclear receptor signaling such as LRH-1 (47-51).

The phenotype of myeloid cell-specific Hdac3 knockout mice is partially resembling the data from Oppi et al. $(41,52)$. Hoeksema et al. showed that Hdac3-deficient mice develop more atherosclerotic plaques in the aortic sinus compared to control mice (52). Furthermore, Hdac3-deficient macrophages displayed an anti-inflammatory wound-healing like phenotype. 
On the other side, atherosclerotic plaques from myeloid cellspecific Hdac3 knockout mice displayed increased collagen disposition and enlarged protective fibrous caps, while the myeloid cell-specific Ncor1 knockout plaques had increased necrotic cores (41).

In line with the data from Oppi et al. (41), Fan et al. showed that myeloid cell-specific Gps2-knockout mice display an increased expression of pro-inflammatory cytokines and chemokines, such as $\mathrm{Ccl} 2$ and $\mathrm{Ccl} 7$, which is characteristic of pro-inflammatory M1-type macrophage activation (53). Moreover, the authors used palmitate as obesity-linked metabolic trigger of inflammation and observed that Gps2deficient macrophages had an elevated pro-inflammatory gene signature (53). Vice-versa, transplantation of Gps2overexpressing bone marrow into two mouse models of obesity reduced inflammation and improved insulin sensitivity in recipient mice (53). Moreover, docking of GPS2 and LXR stimulate H3K9 demethylation on the Abcg1 promoter, thus promoting its expression and mediating cholesterol efflux to HDL particles in monocytic THP-1 cells (54). Contrary to the induction of Abcg1 via LXR, GPS2 interacts with NF- $\kappa B$ to promote the expression of Abcal upon LPS stimulation (55). These elegant studies demonstrate how GPS2 promotes macrophage cholesterol efflux upon different stimuli, i.e. via oxysterol-triggered LXR activation or via LPS-driven NF- $\mathrm{KB}$ activation. Interestingly, the activation of both processes by GPS2 is independent of NCOR1-HDAC3 recruitment $(54,55)$.

Aside from its effects on atherosclerotic plaques and metabolism, macrophage NCOR1 seems to play a pivotal role in the heart. Genetic deletion of Ncor1 in macrophages led to reduced infarct size and improved cardiac function in mice with experimental myocardial infarction (56). These findings were explained by suppression of inflammatory transcriptional programs (interleukin-1 $\beta$, interleukin-6, AP-1 and nuclear factor- $\mathrm{KB}$ ) and reduced macrophage proliferation (due to inhibition of cell cycle progression). Hence, macrophage NCOR1 may act as an upstream regulator of myocardial inflammation thus participating to left ventricular hypertrophy, diastolic dysfunction and microvascular disease, key hallmarks of heart failure (57). In the same study, macrophage NCOR1 deficiency markedly inhibited neointimal hyperplasia and vascular remodeling in a mouse model of arterial wire injury (56). Taken together, these results suggest that selective modulation of NCOR1 in macrophages could have important implications for the prevention of myocardial ischemic damage, heart failure, coronary heart disease and intracoronary stent restenosis. Further molecular work and preclinical studies are needed to better explore the potential of NCOR targeting approaches in cardiovascular disease.

\section{NCOR1 Complex in the Liver}

The liver plays a crucial role in the development of atherosclerosis by regulating metabolic and inflammatory processes, such as the expression of pro-inflammatory cytokines and acute phase response proteins, the secretion of VLDL particles, the uptake of cholesterol from the circulation, and the biliary cholesterol excretion. An immunometabolic dysregulation in the liver can promote nonalcoholic fatty liver disease and the development of atherosclerosis. Importantly, nonalcoholic fatty liver disease leads to adverse cardiovascular functions, such as increased oxidative stress and endothelial dysfunction, hypercoagulability, and accelerated development of atherosclerosis (58-60).

Several studies addressed the physiological functions of NCOR1, NCOR2, and HDAC3 in the liver, which target several lipid-responsive nuclear receptors (Figure 2) (24, 28, $29,61-67)$. The role of NCOR1 in liver energy metabolism is particularly interesting during the fasting-feeding transition: both HDAC3 and NCOR1 are known to repress lipogenic genes, but paradoxically, NCOR1 was also reported to be critical for inhibition of PPAR $\alpha$, hepatic fatty oxidation and ketogenesis $(63,64)$. This is due to the ability of NCOR1 to select its repressor targets in a context-dependent manner to orchestrate liver energy metabolism depending on the energy status of the cell (37). Upon feeding, high levels of glucose and insulin activate the target of rapamycin complex 1 (mTORC1)AKT signaling pathway, thus phosphorylating serine 1460 of NCOR1 (pS1460 NCOR1) (Figure 2). pS1460 decreases the ability of NCOR1 to interact with LXR, thus promoting the transcription of lipogenic LXRs target genes, and conversely, by fostering the interaction with PPAR $\alpha$ and ERR $\alpha$, with subsequent repression of downstream ketogenic and Oxphos genes (37).

Besides being regulated by phosphorylation in the liver, the translation of NCOR1 can also be blocked by the microRNA miR-199a-5p (68) (Figure 2). The authors used a bioinformatic approach to identify miRNAs affected in a non-alcoholic steatohepatitis (NASH) model induced by a methioninecholine-deficient (MCD) diet. The MCD diet increased miR199a-5p expression, which in turn blocks the translation of the Ncor 1 mRNA by binding to a conserved 3' untranslated region (68).

To assess the function of NCOR1 in thyroid hormone receptor (TR) regulation, the group of A. Hollenberg generated a truncated form of NCOR1 which lacks the two main nuclear receptor interacting domains (NCOR1 $\triangle \mathrm{ID})$ and thus, for example, cannot interact with TRs or LXRs $(69,70)$. Interestingly, they found that mice with a disrupted nuclear receptor binding domain show a reduced content of cholesterol in the liver and an increased synthesis of alternative bile acid. In turn, these less hydrophobic bile acids have a lower capacity to bind fats and sterols in the intestine and thus reduce their absorption (38). The increased expression of alternative bile acid synthesis genes is a consequence of TR $\beta 1$ de-repression caused by the mutant NCOR1 (38).

GPS2 exerts protective function in the liver by interacting with SUMOylated nuclear receptors, such as LRH-1 and LXR $\beta$, to repress inflammatory cytokine expression during the hepatic acute phase response (71). On the other side, hepatic deletion of GPS2 reduces nonalcoholic steatohepatitis via induction of PPAR $\alpha$ driven lipid catabolism (67). This repression of PPAR $\alpha$ happens in cooperation with NCOR1, but not NCOR2 (67). Conversely to 
the GPS2 knockouts, hepatic deletion of TBL1 promotes hypertriglyceridemia and hepatic steatosis on both a normal chow or high-fat diet by impairing PPAR $\alpha$-driven lipid catabolism (72). The induction of PPAR $\alpha$ was mediated by increased recruitment of NCOR1-HDAC3 complexes in the absence of TBL1 (72). These data underline that the repressive function and target specificity of the NCOR1 complex is largely dependent on its co-regulators.

\section{NCOR1 in Cardiomyocytes}

Previous studies demonstrated that NCOR1 deficiency increases the activity of MEF2d in skeletal muscle (35). In a recent publication, Li et al. affirmed the suppressive role of NCOR1 in regulating the size of cardiomyocytes, presenting strong evidence of interactions among NCOR1, MEF2, and class IIa HDACs, being MEF2a and MEF2d key transcription factors interceded the impact of NCOR1 on cardiomyocyte size (73). As Li et al. described, NCOR1 may be considered as a stress-responsive and cardioprotective regulator during cardiac hypertrophy, showing that its deficiency led to cardiac hypertrophy under physiological condition and aggravated hypertrophy induced by pressure overload (73).

NCOR1 usually cooperates with HDACs to execute its repressive activities (28). The regulatory paradigm in cardiac hypertrophy involves alterations in gene expression that is mediated by chromatin remodeling. HDACs remove the acetyl group from histones, resulting in its hypoacetylation, which diminishes chromatin accessibility for transcription factors, leading to repression of transcription (74). The Class I HDAC3 enzyme participates in the repressive activities of NCOR1, and its deficiency in cardiomyocytes causes severe cardiac hypertrophy at an early stage. Interestingly, Li et al. identified class IIa to be involved in the process of cardiomyocytes, demonstrating that NCOR1 works more likely through class IIa instead of class I to affect cardiac hypertrophy (73).

\section{BCL6/NCOR1-Mediated Repression of Inflammation}

B cell lymphoma-6 (BCL6) belongs to a class of zinc-finger transcription factors and acts as a transcriptional repressor. It regulates the development of germinal centers, $\mathrm{B}$ and $\mathrm{T}$ cells, coordinates the activation of macrophages, and was described as a proto-oncogene (75). Importantly, the interaction of BCL6 with NCOR complexes is essential to mediate its transrepressive activity in several biological processes $(75,76)$.

The PPAR $\delta$ agonist GW0742 protects against angiotensin II (AngII)-accelerated atherosclerosis by inducing the expression of the $B c l 6$, and the regulators of $G$ protein-coupled signaling (RGS) proteins RGS4 and RGS5, which in turn inhibit the expression of pro-inflammatory and atherogenic genes (77). Interestingly, ChIP-seq data demonstrate that the BCL6 and NCORs cistrome overlap in about $50 \%$ of all DNA binding sites, and that binding sites that are synergistically bound by BCL6 and NCORs are highly enriched for inflammatory and atherogenic $\mathrm{NF}-\kappa \mathrm{B}$ target genes (78).
The deletion of Bcl6 in the liver leads to a derepression of PPAR $\alpha$-driven enzymes mediating fatty acid oxidation and thus protects against high-fat diet-induced hepatic steatosis (79). Interestingly, binding of the corepressors NCOR1, NCOR2 and HDAC3 to BCL6-binding sites was reduced in Bcl6-deficient livers, and these sites displayed increased enhancer/promoter activity as shown by enhanced histone 3 lysine 27 acetylation (H3K27ac) (79). These data suggest that hepatic BCL6 recruits a subset of NCOR/HADC3 complexes to the promoter of specific target genes regulating lipid metabolism.

\section{Potential Functions of the NCOR1 Complex in Endothelial and T Cells}

So far, the role of NCOR1 using an endothelial cell-specific genetic model has not been explored. Therefore, this section is limited to describe the function of its tight cofactor HDAC3 (28). HDAC3 is essential for endothelial monolayer survival and integrity. Studies demonstrated that HDAC3 is engaged in the differentiation of embryonic stem cells into endothelial progenitors and determinant for endothelial cell survival (64). Zampetaki et al. demonstrated that disturbed flow induces transient stabilization of the HDAC3 protein in endothelial cells. It happens through the activation of the VEGFR2 and PI3 kinase signaling pathways (80). HDAC3 expression increases near branch openings compared with areas of high laminar flow, which was also confirmed by exposure of endothelial cells to disturbed flow in vitro (80). It is therefore likely that NCOR1 might be involved in these processes as well.

To obtain a good overview about the function of NCOR1 in T cells we refer to an excellent review from Muller et al. (81). Most studies assessed the role of NCOR1 during T cell development, while only a few focused on its function in mature $\mathrm{T}$ cells in pathophysiological conditions. The lethal phenotype of the complete Ncor1 knockout mice developed by Jepsen et al. demonstrated that constitutive Ncor1-deficiency conferred impaired thymocyte development (33). Further studies using $\mathrm{T}$ cell-specific deletions of Ncor1 in mice revealed that the number of double negative thymocytes and peripheral $\mathrm{T}$ cells are decreased in the absence of NCOR1 $(82,83)$. Recently, it was shown that NCOR1 regulates the function of CD4+ and Th1 cells by regulating the expression of IFN- $\gamma$ (84). Consistently, Zhang et al. showed that deletion of Ncor1 in male SJL T cells disrupted the fenofibrate-driven repression of IFN- $\gamma$ synthesis, possibly also explaining differences in Th1 responses between male and female mice (85). Another interesting report demonstrated that NCOR1 represses the tolerogenic program in dendritic cells. As a consequence, dendritic cell-specific Ncor 1 knockout mice display an increased number of FoxP3+ regulatory $\mathrm{T}$ cells (86). In terms of atherogenesis, this could turn into a protective effect.

\section{PERSPECTIVES}

Research over the past decades identified various molecular factors that affect atherogenesis, including inflammatory and metabolic 
regulators $(2,87,88)$. In the last years, we have learned that immunometabolic pathways are often interconnected, and we are now starting to understand the major factors involved in these immunometabolic mechanisms (10, 11). Targeting immunometabolic pathways, e.g., those regulated by NCOR1, might become an attractive approach to prevent the development of atherosclerosis since they regulate various processes that promote the development of the disease. Moreover, since these pathways are also implicated in the development of other chronic immunometabolic diseases, such as obesity, type 2 diabetes, and nonalcoholic fatty liver disease, their mechanistic dissection will be relevant to understand development and progression of immunometabolic diseases on a broader scale.

Which could be suitable strategies to target NCOR1? One option would be to develop small molecule agonists or antagonists that interfere or facilitate the interaction of NCOR1 to a specific target nuclear receptor. Although this would be a specific approach, it would be very laborious and time-consuming. Another option is offered by current developments in gene therapy (including CRISPR therapeutics) and small interference RNA (siRNA) medicine, which offer excellent future targeting opportunities. The liver is an easily accessible target organ and new emerging drugs using siRNAs demonstrate that transcribed RNA can be targeted in a liverspecific fashion by conjugating the synthetic siRNA to triantennary $\mathrm{N}$-acetylgalactosamine carbohydrates (e.g., as it is

\section{REFERENCES}

1. Weber C, Noels H. Atherosclerosis: current pathogenesis and therapeutic options. Nat Med (2011) 17(11):1410-22. doi: 10.1038/nm.2538

2. Libby P, Ridker PM, Hansson GK. Progress and challenges in translating the biology of atherosclerosis. Nature (2011) 473(7347):317-25. doi: 10.1038/ nature10146

3. Jonsson AL, Backhed F. Role of gut microbiota in atherosclerosis. Nat Rev Cardiol (2017) 14(2):79-87. doi: 10.1038/nrcardio.2016.183

4. Kessler T, Vilne B, Schunkert H. The impact of genome-wide association studies on the pathophysiology and therapy of cardiovascular disease. EMBO Mol Med (2016) 8(7):688-701. doi: 10.15252/emmm.201506174

5. Roberts R. Genetics of coronary artery disease. Circ Res (2014) 114(12):1890903. doi: 10.1161/CIRCRESAHA.114.302692

6. Cannon CP, Blazing MA, Giugliano RP, McCagg A, White JA, Theroux P, et al. Ezetimibe Added to Statin Therapy after Acute Coronary Syndromes. New Engl J Med (2015) 372(25):2387-97. doi: 10.1056/NEJMoa1410489

7. Nissen SE, Tuzcu EM, Schoenhagen P, Crowe T, Sasiela WJ, Tsai J, et al. Statin therapy, LDL cholesterol, C-reactive protein, and coronary artery disease. New Engl J Med (2005) 352(1):29-38. doi: 10.1056/NEJMoa042000

8. Ridker PM, Cannon CP, Morrow D, Rifai N, Rose LM, McCabe CH, et al. Creactive protein levels and outcomes after statin therapy. New Engl J Med (2005) 352(1):20-8. doi: 10.1056/NEJMoa042378

9. Oppi S, Luscher TF, Stein S. Mouse Models for Atherosclerosis ResearchWhich Is My Line? Front Cardiovasc Med (2019) 6:46(46):46. doi: 10.3389/ fcvm.2019.00046

10. Hotamisligil GS. Foundations of Immunometabolism and Implications for Metabolic Health and Disease. Immunity (2017) 47(3):406-20. doi: 10.1016/ j.immuni.2017.08.009

11. Lee YS, Wollam J, Olefsky JM. An Integrated View of Immunometabolism. Cell (2018) 172(1-2):22-40. doi: 10.1016/j.cell.2017.12.025

12. Saltiel AR, Olefsky JM. Inflammatory mechanisms linking obesity and metabolic disease. J Clin Invest (2017) 127(1):1-4. doi: 10.1172/JCI92035. used to target PCSK9 in the liver $(89,90))$. However, specifically targeting other organs or cells, such as cardiomyocytes and macrophages, continues to be challenging. Moreover, the identification of atherogenic targets, such as CD36 in macrophages (41), might lead to the identification of druggable targets and hence to the development of new therapeutic strategies to treat atherosclerotic disease patients that are at a very high risk to suffer a transient ischemic attack, stroke or acute coronary syndrome.

\section{AUTHOR CONTRIBUTIONS}

All authors analyzed the literature and wrote the manuscript. All authors contributed to the article and approved the submitted version.

\section{FUNDING}

SS was supported by the Swiss National Science Foundation (PZOOP3_161521), the SwissLife Jubiläumsstiftung, the Swiss Heart Foundation (FF19025), and the Foundation for Research in Science and the Humanities at the University of Zurich (STWF-20-008). treatment of metabolic disease. J.M. Olefsky owns stock in Catabasis Pharmaceuticals and receives consulting income from Cymabay Inc., Second Genome, and AntriaBio.

13. Margueron R, Reinberg D. Chromatin structure and the inheritance of epigenetic information. Nat Rev Genet (2010) 11(4):285-96. doi: 10.1038/ nrg2752

14. Smith E, Shilatifard A. The chromatin signaling pathway: diverse mechanisms of recruitment of histone-modifying enzymes and varied biological outcomes. Mol Cell (2010) 40(5):689-701. doi: 10.1016/j.molcel.2010.11.031

15. Mouchiroud L, Eichner LJ, Shaw RJ, Auwerx J. Transcriptional coregulators: fine-tuning metabolism. Cell Metab (2014) 20(1):26-40. doi: 10.1016/ j.cmet.2014.03.027

16. Costantino S, Mohammed SA, Ambrosini S, Paneni F. Epigenetic processing in cardiometabolic disease. Atherosclerosis (2019) 281:150-8. doi: 10.1016/ j.atherosclerosis.2018.09.029

17. Glass CK, Ogawa S. Combinatorial roles of nuclear receptors in inflammation and immunity. Nat Rev Immunol (2006) 6(1):44-55. doi: 10.1038/nri1748

18. Francis GA, Fayard E, Picard F, Auwerx J. Nuclear receptors and the control of metabolism. Annu Rev Physiol (2003) 65:261-311. doi: 10.1146/ annurev.physiol.65.092101.142528

19. Kurakula K, Hamers AA, de Waard V, de Vries CJ. Nuclear Receptors in atherosclerosis: a superfamily with many 'Goodfellas'. Mol Cell Endocrinol (2013) 368(1-2):71-84. doi: 10.1016/j.mce.2012.05.014

20. Ghisletti S, Huang W, Ogawa S, Pascual G, Lin ME, Willson TM, et al. Parallel SUMOylation-dependent pathways mediate gene- and signal-specific transrepression by LXRs and PPARgamma. Mol Cell (2007) 25(1):57-70. doi: 10.1016/j.molcel.2006.11.022

21. Ghisletti S, Huang W, Jepsen K, Benner C, Hardiman G, Rosenfeld MG, et al. Cooperative NCoR/SMRT interactions establish a corepressor-based strategy for integration of inflammatory and anti-inflammatory signaling pathways. Genes Dev (2009) 23(6):681-93. doi: 10.1101/gad.1773109

22. Pascual G, Fong AL, Ogawa S, Gamliel A, Li AC, Perissi V, et al. A SUMOylation-dependent pathway mediates transrepression of inflammatory 
response genes by PPAR-gamma. Nature (2005) 437(7059):759-63. doi: 10.1038 /nature 03988

23. Glass CK, Saijo K. Nuclear receptor transrepression pathways that regulate inflammation in macrophages and T cells. Nat Rev Immunol (2010) 10 (5):365-76. doi: $10.1038 /$ nri2748

24. Treuter E, Venteclef N. Transcriptional control of metabolic and inflammatory pathways by nuclear receptor SUMOylation. Biochim Biophys Acta (2011) 1812(8):909-18. doi: 10.1016/j.bbadis.2010.12.008

25. Venteclef N, Jakobsson T, Steffensen KR, Treuter E. Metabolic nuclear receptor signaling and the inflammatory acute phase response. Trends Endocrinol Metabol: TEM (2011) 22(8):333-43. doi: 10.1016/j.tem.2011.04.004

26. Zhang HM, Chen H, Liu W, Liu H, Gong J, Wang H, et al. AnimalTFDB: a comprehensive animal transcription factor database. Nucleic Acids Res (2012) 40(Database issue):D144-9. doi: 10.1093/nar/gkr965

27. Perissi V, Jepsen K, Glass CK, Rosenfeld MG. Deconstructing repression: evolving models of co-repressor action. Nat Rev Genet (2010) 11(2):109-23. doi: $10.1038 / \mathrm{nrg} 2736$

28. Mottis A, Mouchiroud L, Auwerx J. Emerging roles of the corepressors NCoR1 and SMRT in homeostasis. Genes Dev (2013) 27(8):819-35. doi: 10.1101/gad.214023.113

29. Emmett MJ, Lazar MA. Integrative regulation of physiology by histone deacetylase 3. Nat Rev Mol Cell Biol (2019) 20(2):102-15. doi: 10.1038/ s41580-018-0076-0

30. Liang N, Jakobsson T, Fan R, Treuter E. The Nuclear Receptor-Co-repressor Complex in Control of Liver Metabolism and Disease. Front Endocrinol (2019) 10:411. doi: 10.3389/fendo.2019.00411

31. Neele AE, Van den Bossche J, Hoeksema MA, de Winther MP. Epigenetic pathways in macrophages emerge as novel targets in atherosclerosis. Eur $J$ Pharmacol (2015) 763(Pt A):79-89. doi: 10.1016/j.ejphar.2015.03.101

32. Jepsen K, Solum D, Zhou T, McEvilly RJ, Kim HJ, Glass CK, et al. SMRTmediated repression of an $\mathrm{H} 3 \mathrm{~K} 27$ demethylase in progression from neural stem cell to neuron. Nature (2007) 450:415-9. doi: 10.1038/nature06270

33. Jepsen K, Hermanson O, Onami TM, Gleiberman AS, Lunyak V, McEvilly RJ, et al. Combinatorial roles of the nuclear receptor corepressor in transcription and development. Cell (2000) 102(6):753-63. doi: 10.1016/S0092-8674(00) 00064-7

34. Jepsen K, Gleiberman AS, Shi C, Simon DI, Rosenfeld MG. Cooperative regulation in development by SMRT and FOXP1. Genes Dev (2008) 22 (6):740-5. doi: 10.1101/gad.1637108. doi: 22/6/740 [pii].

35. Yamamoto H, Williams EG, Mouchiroud L, Canto C, Fan W, Downes M, et al. NCoR1 is a conserved physiological modulator of muscle mass and oxidative function. Cell (2011) 147(4):827-39. doi: 10.1016/j.cell.2011. 10.017

36. Li P, Fan W, Xu J, Lu M, Yamamoto H, Auwerx J, et al. Adipocyte NCoR knockout decreases PPARgamma phosphorylation and enhances PPARgamma activity and insulin sensitivity. Cell (2011) 147(4):815-26. doi: 10.1016/j.cell.2011.09.050

37. Jo YS, Ryu D, Maida A, Wang X, Evans RM, Schoonjans K, et al. Phosphorylation of the nuclear receptor corepressor 1 by protein kinase $\mathrm{B}$ switches its corepressor targets in the liver in mice. Hepatology (2015) 62 (5):1606-18. doi: 10.1002/hep.27907

38. Astapova I, Ramadoss P, Costa-e-Sousa RH, Ye F, Holtz KA, Li Y, et al. Hepatic nuclear corepressor 1 regulates cholesterol absorption through a TRbetal-governed pathway. J Clin Invest (2014) 124(5):1976-86. doi: 10.1172/JCI73419

39. Li P, Spann NJ, Kaikkonen MU, Lu M, Oh da Y, Fox JN, et al. NCoR repression of LXRs restricts macrophage biosynthesis of insulin-sensitizing omega 3 fatty acids. Cell (2013) 155(1):200-14. doi: 10.1016/j.cell.2013.08.054

40. Wiesner P, Choi SH, Almazan F, Benner C, Huang W, Diehl CJ, et al. Low doses of lipopolysaccharide and minimally oxidized low-density lipoprotein cooperatively activate macrophages via nuclear factor kappa B and activator protein-1: possible mechanism for acceleration of atherosclerosis by subclinical endotoxemia. Circ Res (2010) 107(1):56-65. doi: 10.1161/ CIRCRESAHA.110.218420

41. Oppi S, Nusser-Stein S, Blyszczuk P, Wang X, Jomard A, Marzolla V, et al. Macrophage NCOR1 protects from atherosclerosis by repressing a proatherogenic PPARgamma signature. Eur Heart J (2020) 41(9):995-1005. doi: 10.1093/eurheartj/ehz667
42. Moore KJ, Tabas I. Macrophages in the pathogenesis of atherosclerosis. Cell (2011) 145(3):341-55. doi: 10.1016/j.cell.2011.04.005

43. Swirski FK, Nahrendorf M. Leukocyte behavior in atherosclerosis, myocardial infarction, and heart failure. Science (2013) 339(6116):161-6. doi: 10.1126/ science. 1230719

44. Ogawa S, Lozach J, Jepsen K, Sawka-Verhelle D, Perissi V, Sasik R, et al. A nuclear receptor corepressor transcriptional checkpoint controlling activator protein 1-dependent gene networks required for macrophage activation. Proc Natl Acad Sci U.S.A. (2004) 101(40):14461-6. doi: 10.1073/pnas.0405786101

45. Perissi V, Aggarwal A, Glass CK, Rose DW. Rosenfeld MG. A corepressor/ coactivator exchange complex required for transcriptional activation by nuclear receptors and other regulated transcription factors. Cell (2004) 116 (4):511-26. doi: 10.1016/S0092-8674(04)00133-3

46. Emini Veseli B, Perrotta P, De Meyer GRA, Roth L, Van der Donckt C, Martinet W, et al. Animal models of atherosclerosis. Eur J Pharmacol (2017) 816:3-13. doi: 10.1016/j.ejphar.2017.05.010

47. Lefevre L, Authier H, Stein S, Majorel C, Couderc B, Dardenne C, et al. LRH-1 mediates anti-inflammatory and antifungal phenotype of IL-13-activated macrophages through the PPARgamma ligand synthesis. Nat Commun (2015) 6:6801. doi: 10.1038/ncomms7801

48. Miranda DA, Krause WC, Cazenave-Gassiot A, Suzawa M, Escusa H, Foo JC, et al. LRH-1 regulates hepatic lipid homeostasis and maintains arachidonoyl phospholipid pools critical for phospholipid diversity. JCI Insight (2018) 3(5). doi: 10.1172/jci.insight.96151

49. Stein S, Lemos V, Xu P, Demagny H, Wang X, Ryu D, et al. Impaired SUMOylation of nuclear receptor LRH-1 promotes nonalcoholic fatty liver disease. J Clin Invest (2017) 127(2):583-92. doi: 10.1172/JCI85499

50. Stein S, Oosterveer MH, Mataki C, Xu P, Lemos V, Havinga R, et al. SUMOylation-dependent LRH-1/PROX1 interaction promotes atherosclerosis by decreasing hepatic reverse cholesterol transport. Cell Metab (2014) 20(4):603-13. doi: 10.1016/j.cmet.2014.07.023

51. Xu P, Oosterveer MH, Stein S, Demagny H, Ryu D, Moullan N, et al. LRH-1dependent programming of mitochondrial glutamine processing drives liver cancer. Genes Dev (2016) 30(11):1255-60. doi: 10.1101/gad.277483.116

52. Hoeksema MA, Gijbels MJ, Van den Bossche J, van der Velden S, Sijm A, Neele AE, et al. Targeting macrophage Histone deacetylase 3 stabilizes atherosclerotic lesions. EMBO Mol Med (2014) 6(9):1124-32. doi: 10.15252/ emmm. 201404170

53. Fan R, Toubal A, Goni S, Drareni K, Huang Z, Alzaid F, et al. Loss of the corepressor GPS2 sensitizes macrophage activation upon metabolic stress induced by obesity and type 2 diabetes. Nat Med (2016) 22(7):780-91. doi: 10.1038/nm.4114

54. Jakobsson T, Venteclef N, Toresson G, Damdimopoulos AE, Ehrlund A, Lou $\mathrm{X}$, et al. GPS2 is required for cholesterol efflux by triggering histone demethylation, LXR recruitment, and coregulator assembly at the ABCG1 locus. Mol Cell (2009) 34(4):510-8. doi: 10.1016/j.molcel.2009.05.006

55. Huang Z, Liang N, Damdimopoulos A, Fan R, Treuter E. G protein pathway suppressor 2 (GPS2) links inflammation and cholesterol efflux by controlling lipopolysaccharide-induced ATP-binding cassette transporter A1 expression in macrophages. FASEB J (2019) 33(2):1631-43. doi: 10.1096/fj.201801123R

56. Du LJ, Sun JY, Zhang WC, Wang YL, Zhu H, Liu T, et al. Macrophage NCOR1 Deficiency Ameliorates Myocardial Infarction and Neointimal Hyperplasia in Mice. J Am Heart Assoc (2020) 9(15):e015862. doi: 10.1161/JAHA.120.015862

57. Metra M, Teerlink JR. Heart failure. Lancet (2017) 390(10106):1981-95. doi: 10.1016/S0140-6736(17)31071-1

58. Anstee QM, Targher G, Day CP. Progression of NAFLD to diabetes mellitus, cardiovascular disease or cirrhosis. Nat Rev Gastroenterol Hepatol (2013) 10 (6):330-44. doi: 10.1038/nrgastro.2013.41

59. Byrne CD, Targher G. NAFLD: a multisystem disease. J Hepatol (2015) 62(1 Suppl):S47-64. doi: 10.1016/j.jhep.2014.12.012

60. Bhatia LS, Curzen NP, Calder PC, Byrne CD. Non-alcoholic fatty liver disease: a new and important cardiovascular risk factor? Eur Heart J (2012) 33 (10):1190-200. doi: 10.1093/eurheartj/ehr453

61. Shimizu H, Astapova I, Ye F, Bilban M, Cohen RN, Hollenberg AN. NCoR1 and SMRT play unique roles in thyroid hormone action in vivo. Mol Cell Biol (2015) 35(3):555-65. doi: 10.1128/MCB.01208-14

62. Jeyakumar M, Liu XF, Erdjument-Bromage H, Tempst P, Bagchi MK. Phosphorylation of thyroid hormone receptor-associated nuclear receptor corepressor holocomplex by the DNA-dependent protein kinase enhances its 
histone deacetylase activity. J Biol Chem (2007) 282(13):9312-22. doi: 10.1074/jbc.M609009200

63. Sun Z, Feng D, Fang B, Mullican SE, You SH, Lim HW, et al. Deacetylaseindependent function of HDAC3 in transcription and metabolism requires nuclear receptor corepressor. Mol Cell (2013) 52(6):769-82. doi: 10.1016/ j.molcel.2013.10.022

64. Alenghat T, Meyers K, Mullican SE, Leitner K, Adeniji-Adele A, Avila J, et al. Nuclear receptor corepressor and histone deacetylase 3 govern circadian metabolic physiology. Nature (2008) 456(7224):997-1000. doi: 10.1038/nature07541

65. Feng D, Liu T, Sun Z, Bugge A, Mullican SE, Alenghat T, et al. A circadian rhythm orchestrated by histone deacetylase 3 controls hepatic lipid metabolism. Science (2011) 331(6022):1315-9. doi: 10.1126/science.1198125

66. Zhang J, Xu Z, Gu J, Jiang S, Liu Q, Zheng Y, et al. HDAC3 inhibition in diabetic mice may activate Nrf2 preventing diabetes-induced liver damage and FGF21 synthesis and secretion leading to aortic protection. Am J Physiol Endocrinol Metab (2018) 315(2):E150-E62. doi: 10.1152/ajpendo.00465.2017

67. Liang N, Damdimopoulos A, Goni S, Huang Z, Vedin LL, Jakobsson T, et al. Hepatocyte-specific loss of GPS2 in mice reduces non-alcoholic steatohepatitis via activation of PPARalpha. Nat Commun (2019) 10 (1):1684. doi: 10.1038/s41467-019-09524-z

68. Zhang B, Wang R, Du J, Niu J, Zhang R, Xu S, et al. Upregulated microRNA199a-5p inhibits nuclear receptor corepressor 1 translation in mice with nonalcoholic steatohepatitis. Mol Med Rep (2014) 10(6):3080-6. doi: 10.3892/ mmr.2014.2592

69. Astapova I, Lee LJ, Morales C, Tauber S, Bilban M, Hollenberg AN. The nuclear corepressor, NCoR, regulates thyroid hormone action in vivo. Proc Natl Acad Sci U.S.A. (2008) 105(49):19544-9. doi: 10.1073/pnas.0804604105

70. Vella KR, Hollenberg AN. The actions of thyroid hormone signaling in the nucleus. Mol Cell Endocrinol (2017) 458:127-35. doi: 10.1016/ j.mce.2017.03.001

71. Venteclef N, Jakobsson T, Ehrlund A, Damdimopoulos A, Mikkonen L, Ellis E, et al. GPS2-dependent corepressor/SUMO pathways govern anti-inflammatory actions of LRH-1 and LXRbeta in the hepatic acute phase response. Genes Dev (2010) 24(4):381-95. doi: 10.1101/gad.545110. doi: 24/4/381 [pii].

72. Kulozik P, Jones A, Mattijssen F, Rose AJ, Reimann A, Strzoda D, et al. Hepatic deficiency in transcriptional cofactor TBL1 promotes liver steatosis and hypertriglyceridemia. Cell Metab (2011) 13(4):389-400. doi: 10.1016/ j.cmet.2011.02.011

73. Li C, Sun XN, Chen BY, Zeng MR, Du LJ, Liu T, et al. Nuclear receptor corepressor 1 represses cardiac hypertrophy. EMBO Mol Med (2019) 11(11): e9127. doi: 10.15252/emmm.201809127

74. de Ruijter AJ, van Gennip AH, Caron HN, Kemp S, van Kuilenburg AB. Histone deacetylases (HDACs): characterization of the classical HDAC family. Biochem J (2003) 370(Pt 3):737-49. doi: 10.1042/BJ20021321

75. Cardenas MG, Oswald E, Yu W, Xue F, MacKerell ADJr., Melnick AM. The Expanding Role of the BCL6 Oncoprotein as a Cancer Therapeutic Target. Clin Cancer Res Off J Am Assoc Cancer Res (2017) 23(4):885-93. doi: 10.1158/ 1078-0432.CCR-16-2071

76. Huynh KD, Bardwell VJ. The BCL-6 POZ domain and other POZ domains interact with the co-repressors N-CoR and SMRT. Oncogene (1998) 17 (19):2473-84. doi: 10.1038/sj.onc.1202197

77. Takata Y, Liu J, Yin F, Collins AR, Lyon CJ, Lee CH, et al. PPARdeltamediated antiinflammatory mechanisms inhibit angiotensin II-accelerated atherosclerosis. Proc Natl Acad Sci U.S.A. (2008) 105(11):4277-82. doi: 10.1073/pnas.0708647105
78. Barish GD, Yu RT, Karunasiri MS, Becerra D, Kim J, Tseng TW, et al. The Bcl6SMRT/NCoR cistrome represses inflammation to attenuate atherosclerosis. Cell Metab (2012) 15(4):554-62. doi: 10.1016/j.cmet.2012.02.012

79. Sommars MA, Ramachandran K, Senagolage MD, Futtner CR, Germain DM, Allred AL, et al. Dynamic repression by BCL6 controls the genomewide liver response to fasting and steatosis. eLife (2019) 8. doi: 10.7554/ eLife. 43922

80. Zampetaki A, Zeng L, Margariti A, Xiao Q, Li H, Zhang Z, et al. Histone deacetylase 3 is critical in endothelial survival and atherosclerosis development in response to disturbed flow. Circulation (2010) 121(1):13242. doi: 10.1161/CIRCULATIONAHA.109.890491

81. Muller L, Hainberger D, Stolz V, Ellmeier W. NCOR1-a new player on the field of T cell development. J Leukoc Biol (2018) 104(6):1061-8. doi: 10.1002/ JLB.1RI0418-168R

82. Muller L, Hainberger D, Stolz V, Hamminger P, Hassan H, Preglej T, et al. The corepressor NCOR1 regulates the survival of single-positive thymocytes. Sci Rep (2017) 7(1):15928. doi: 10.1038/s41598-017-15918-0

83. Wang J, He N, Zhang N, Quan D, Zhang S, Zhang C, et al. NCoR1 restrains thymic negative selection by repressing Bim expression to spare thymocytes undergoing positive selection. Nat Commun (2017) 8(1):959. doi: 10.1038/ s41467-017-00931-8

84. Hainberger D, Stolz V, Zhu C, Schuster M, Muller L, Hamminger P, et al. NCOR1 Orchestrates Transcriptional Landscapes and Effector Functions of CD4 (+) T Cells. Front Immunol (2020) 11:579:579. doi: 10.3389/fimmu.2020.00579

85. Zhang MA, Ahn JJ, Zhao FL, Selvanantham T, Mallevaey T, Stock N, et al. Antagonizing Peroxisome Proliferator-Activated Receptor alpha Activity Selectively Enhances Th1 Immunity in Male Mice. J Immunol (2015) 195 (11):5189-202. doi: 10.4049/jimmunol.1500449

86. Ahad A, Stevanin M, Smita S, Mishra GP, Gupta D, Waszak S, et al. NCoR1: Putting the Brakes on the Dendritic Cell Immune Tolerance. iScience (2019) 19:996-1011. doi: 10.1016/j.isci.2019.08.024

87. Hopkins PN. Molecular biology of atherosclerosis. Physiol Rev (2013) 93 (3):1317-542. doi: 10.1152/physrev.00004.2012

88. Kolodgie FD, Yahagi K, Mori H, Romero ME, Trout HHR, Finn AV, et al. High-risk carotid plaque: lessons learned from histopathology. Semin Vasc Surg (2017) 30(1):31-43. doi: 10.1053/j.semvascsurg.2017.04.008

89. Ray KK, Landmesser U, Leiter LA, Kallend D, Dufour R, Karakas M, et al. Inclisiran in Patients at High Cardiovascular Risk with Elevated LDL Cholesterol. New Engl J Med (2017) 376(15):1430-40. doi: 10.1056/ NEJMoa 1615758

90. Fitzgerald K, White S, Borodovsky A, Bettencourt BR, Strahs A, Clausen V, et al. A Highly Durable RNAi Therapeutic Inhibitor of PCSK9. New Engl J Med (2017) 376(1):41-51. doi: 10.1056/NEJMoa1609243

Conflict of Interest: The authors declare that the research was conducted in the absence of any commercial or financial relationships that could be construed as a potential conflict of interest.

Copyright $\odot 2020$ Geiger, Guillaumon, Paneni, Matter and Stein. This is an openaccess article distributed under the terms of the Creative Commons Attribution License (CC BY). The use, distribution or reproduction in other forums is permitted, provided the original author(s) and the copyright owner(s) are credited and that the original publication in this journal is cited, in accordance with accepted academic practice. No use, distribution or reproduction is permitted which does not comply with these terms. 